\title{
GENERALIZED EIGENFUNCTIONS AND REAL AXIS LIMITS OF THE RESOLVENT
}

\author{
BY
}

\author{
N. A. DERZKO
}

ABSTRACT. Let $(\mathcal{H},(.,)$.$) be a Hilbert space and A, E$ be a selfadjoint operator and corresponding spectral measure in $\mathcal{H}\left(A=\int \lambda E(d \lambda)\right)$. It is known that for a suitable positive subspace $\mathcal{H}+\mathcal{H}$ and measure $\rho$ the generalized eigenfunctions

$$
\phi_{\lambda, f}=\lim _{b \rightarrow 0} \frac{E(\boldsymbol{\lambda}-b, \lambda+b]) f}{\rho(\lambda-b, \lambda+b])} \equiv \lim _{\Delta \rightarrow \lambda} \frac{E(\Delta) f}{\rho(\Delta)}
$$

exist in $\mathcal{H}_{-}$, the corresponding negative space, for $\rho$-almost every $\lambda$ and $f \in \mathcal{H}_{+-}$It is shown that for each $\lambda$ the $\phi_{\lambda_{0} f}$ form a pre-Hilbert space $\mathcal{H}_{\lambda}$ using the natural inner product $\left(\phi_{f}, \phi_{g}\right)_{\lambda}=\lim _{\Delta \rightarrow \lambda}((E(\Delta) f, g) / \rho(\Delta))$, and that $\|\phi\|-\leq C\|\phi\|_{\lambda^{\cdot}}$. Furthermore, if $\{\phi(\lambda, a)\}$ is a suitably chosen basis for $\mathcal{H}_{\lambda}$, $-\infty<\bar{\lambda}<\infty$, then one obtains the eigenfunction expansion suggested by

$$
(f, g)=\int \rho(d \boldsymbol{\lambda}) \sum_{\alpha, \beta}(f, \phi(\boldsymbol{\lambda}, \alpha)) \sigma_{\alpha \beta}(\boldsymbol{\lambda}) \overline{(g, \phi(\lambda, \beta))} .
$$

Finally it is shown that, for a suitable function $w(\epsilon, \lambda), \phi_{\lambda, f}$ is given by $\lim _{\epsilon\rfloor 0} w(\epsilon, \lambda)[R(\lambda-i \epsilon) \sim R(\lambda+i \epsilon)] f$, where $R(z)=(z-A)^{-1}$.

Let $\mathcal{H}$ be any Hilbert space and $A, E$ be a selfadjoint operator and corresponding spectral measure in $\mathcal{H}\left(A=\int \lambda E(d \lambda)\right)$. Our two major results involve showing that suitably defined generalized eigenfunctions $\psi(\lambda)$ at each fixed $\lambda$ form a Hilbert space using a natural inner product. Furthermore, a basis for this space can be obtained by differentiating $E()$.$f at the point \lambda$ with respect to a suitable measure for certain $f \in \mathcal{H}$ or, more usefully, by computing $\lim _{\epsilon 10} w(\epsilon, \lambda)[R(\lambda-i \epsilon)-R(\lambda+i \epsilon)] f$ where $w(\epsilon, \lambda)$ is a suitable weight function which can be taken to be 1 on the absolutely continuous spectrum of $A$ [13, p. 516].

A natural approach in finding an eigenfunction expansion of a selfadjoint extension of a differential operator $P$ involves choosing a family of solutions $\left\{u_{\lambda, a}\right\}$ to

$$
P u=\lambda u \quad(\lambda \text { real })
$$

Received by the editors July 16,1970 .

AMS (MOS) subject classifications (1969). Primary 4730; Secondary 8147, 4765. 
and letting $u_{\lambda, a}(x)$ be the kernel of an integral operator which gives the eigenfunction or Fourier expansion for the problem.

Such a program has been carried out in the case of a formally selfadjoint ordinary differential operator. We here state a shortened version of the TitchmarshKodaira Theorem [7, p. 1364] to indicate the direction in which we are going.

Let $r$ be a formally selfadjoint ordinary differential operator on the interval $I$ (possibly the whole line) and let $T$ be a selfadjoint extension of $r$. Let $U$ be an open subset of the complex plane containing the interval $I$. Let $\left\{\sigma_{k}: k=1, \cdots, n\right\}$ be a basis for the solutions to $\tau \sigma=\lambda \sigma$ such that each $\sigma_{j}$ is continuous on $I \times U$ and analytic on $U$ for fixed $t$. Then there exist measures $\rho_{i j}$ such that

$$
(V f)_{j}(\lambda)=s-\lim \int_{J \uparrow l} f(t) \sigma_{j}(t, \lambda) d t
$$

defines an isometry from $L_{2}(I)$ into $L_{2}\left(\left\{\rho_{i j}\right\}\right)$; that is

$$
\int_{l} f(t) \overline{g(t)} d t=\int_{-\infty}^{\infty}\left[\sum_{i, j=1}^{n}(V f)_{i}(\lambda) \rho_{i j}(d \lambda) \overline{(V g)_{j}(\lambda)}\right] .
$$

Such a theorem is not easily obtainable in the case of a partial differential operator $P$ because of the difficulty in picking a basis for solutions to $P \sigma=\lambda \sigma$ or in even finding a useful topology for the vector space of solutions to $P \sigma=\lambda \sigma$. Furthermore, though it is clear that not all solutions to $P \sigma=\lambda \sigma$ can be used in the eigenfunction expansion (consider $P=-\Delta$ on $E^{2}$ and $\sigma=e^{i p \cdot x}$ with $p=$ $\left(p_{1}, p_{2}\right)$ nonreal and $\left.\sqrt{p_{1}^{2}+p_{2}^{2}}=\lambda>0\right)$, the whole question of eliminating the unwanted ones in the case of infinite domains by specifying a boundary condition at infinity (radiation condition) has only been settled in certain cases [17].

The approach which has succeeded in the case of partial differential operators starts with the spectral measure $E(\cdot)$ arising in the spectral representation of $P$. One then shows that

$$
E(J) f(x)=\int\left[\int_{J} \theta(x, y, \lambda) \tau(d \lambda)\right] f(y) d y
$$

where $J$ is an interval, $\theta$ is a hermitian kernel which satisfies the equation $P \theta(x, \cdot \lambda)=\lambda \theta(x, \cdot, \lambda)$ and $\tau$ is a positive measure on the real line [9].

This kind of result has been generalized to an abstract setting using Hilbert spaces with negative norm by the Russian school. Their work is discussed in a now translated book by Berezanskir [1]. The present paper draws heavily on the theory in Berezanskir's book.

1. Spaces with negative norm $[1$, Chapter 1$]$. Let $\left(\mathcal{H}_{0},(\cdot, \cdot)_{0},\|\cdot\|_{0}\right)$ be a separable Hilbert space containing a dense linear manifold $\mathcal{H}_{+}$which is also a 
Hilbert space under the norm $\|\cdot\|_{+} \geq\|\cdot\|_{0} \cdot$ Then, it can be shown that there exists an operator $T: \mathcal{H}_{0} \rightarrow \mathcal{H}_{+}$such that $(f, g)_{0}=(T f, T g)_{+}$. Furthermore, if we consider the linear functionals $\phi: \mathcal{H}_{+} \rightarrow \mathrm{C},|\phi(f)| \leq\|f\|_{+}$, we find they form a Hilbert space which is the closure of $\mathcal{H}_{0}$ with respect to the inner product $(f, g)_{-}=$ $(T f, T g)_{0}$. We call this Hilbert space $\mathcal{H}_{-}$- hence, the name "space with negative norm". The case of special interest to us occurs when $T$ is a Hilbert-Schmidt operator in $\mathcal{H}_{0}$; that is $\Sigma_{m, n}\left|\left(T e_{n}, e_{m}\right)\right|^{2}<\infty$ for one and therefore for all c.o.n.s. (complete orthonormal sets) $\left\{e_{n}\right\}_{n=1}^{\infty}$ in $\mathcal{H}_{0}$.

We shall denote the trace $\Sigma_{n}\left(S e_{n}, e_{n}\right)$ of a nonnegative operator $S$, whenever it exists, by $\operatorname{tr}(S)$.

2. The Hilbert space of generalized eigenfunctions. Suppose $E(\cdot)$ is a spectral measure and $T$, considered as a mapping of $\mathcal{H}_{0}$ into itself, is a one-toone Hilbert-Schmidt contraction. Then the measure $\rho_{0}$, defined by

$$
\rho_{0}(\Delta)=\operatorname{tr}\left(T^{*} E(\Delta) T\right)
$$

is a bounded nonnegative regular Borel measure.

Given any two nonnegative complete regular Borel measures $\sigma, \rho$ on $\mathbf{R}$ which are bounded on compact sets, using a theorem of de Guzmán [11, Theorem 2.4], one can prove that

$$
\lim _{b \rightarrow 0^{+}} \frac{\sigma([\lambda-b, \lambda+b])}{\rho([\lambda-b, \lambda+b])}
$$

exists $\rho$-a.e. $\lambda$ and yields essentially the Radon-Nikodym derivative $(d \sigma / d \rho)(\lambda)$. To simplify notation, we shall henceforth denote such limits by

$$
\lim _{\Delta \rightarrow \lambda} \frac{\sigma(\Delta)}{\rho(\Delta)}
$$

and assume our measures are complete unless stated otherwise. Also, wk $\lim _{\alpha \rightarrow \beta} \phi_{\alpha}$ $=\psi$ will mean $\lim _{a \rightarrow \beta} \phi_{a}(g)=\psi(g) \forall g \in \mathcal{H}_{+}$, where $\phi_{a}, \psi \in \mathcal{H}_{-}$.

Our first theorem is a slight generalization of a result which is essentially known.

Theorem 1. Let $\rho$ be a locally bounded positive Borel measure. Then

$$
\underset{\Delta \rightarrow \lambda}{\operatorname{wk}} \lim _{\Delta(\Delta)} \frac{E(\Delta)}{\rho(\Delta)}
$$

exists $\rho$-a.e. for all $f \in \mathcal{H}_{+}$yielding a functional $\phi_{\lambda, f} \in \mathcal{H}_{-}$. Furthermore,

$$
\left\|\phi_{\lambda, f_{-}}\right\|_{-} \leq\left(d \rho_{0} / d \rho\right)(\lambda)\|f\|_{+} \rho \text {-a.e. }
$$

and for each $\lambda$ where both $d \rho_{0} / d \rho$ and $d E(\cdot) f / d \rho_{0}$ exist we have 


$$
\frac{d E(\cdot) f}{d \rho}=\frac{d \rho_{0}}{d \rho} \frac{d E(\cdot) f}{d \rho_{0}} .
$$

If $f, g \in \mathcal{H}_{+}$then $(E(\cdot) f, g)$ is absolutely continuous with respect to $\rho_{0} \cdot$

Proof. We first prove the theorem for $\rho=\rho_{0}$.

Let $\mathbb{M}$ be a countable dense subset of $\mathcal{H}_{+}$. Then if $f, g \in \mathbb{M}$ we have

$$
\begin{aligned}
& |(E(\Delta) f, g)|=\left|\left(E(\Delta) T T^{-1} f, T T^{-1} g\right)\right|=\left|\left(T^{*} E(\Delta) T T^{-1} f, T^{-1} g\right)\right| \\
& \quad \leq\left(T^{*} E(\Delta) T T^{-1} f, T^{-1} f\right)^{1 / 2}\left(T^{*} E(\Delta) T T^{-1} g, T^{-1} g\right)^{1 / 2} \leq \rho_{0}(\Delta)\|f\|_{+}\|g\|_{+} \cdot
\end{aligned}
$$

The last inequality is a consequence of the fact that

$$
\rho_{0}(\Delta)=\sum_{k=1}^{\infty}\left(T^{*} E(\Delta) T e_{k}, e_{k}\right) \text { for c.o.n.s. }\left\{e_{k}\right\}
$$

and $T^{-1} f /\left\|T^{-1} f\right\|_{0}=T^{-1} f /\|f\|_{+}$could be taken as $e_{1}$. A similar argument applies to $g$. This means, of course, that $(E(\cdot) /, g)$ is absolutely continuous with respect to $\rho_{0}$. Furthermore, the derivative $d(E(\cdot) /, g) / d \rho_{0}$ exists $\rho_{0}$-a.e. We now make use of the countability of $M$ to exclude a set $\Delta_{0}$ of measure zero and still preserve the existence of $d(E(\cdot) /, g) / d \rho_{0} \forall /, g \in \mathbb{M}$ when $\lambda \notin \Delta_{0}$.

Finally, we use the inequality $|(E(\Delta) f, g)| \leq \rho(\Delta)\|f\|_{+}\|g\|_{+}$to deduce that the limit exists for all $f, g \in \mathcal{H}_{+}$and all $\lambda \notin \Delta_{0}$. The same inequality gives us the result that

$$
\underset{\Delta \rightarrow \lambda}{\operatorname{wk} \lim _{\Delta \rightarrow \lambda}} \frac{E(\Delta) f}{\rho(\Delta)}=\phi_{\lambda, f} \in \mathcal{H}_{-},
$$

and also that $\left\|\phi_{\lambda, f}\right\|_{-} \leq\|f\|_{+}$.

Now if $\rho$ is any nonnegative $\sigma$-finite Borel measure we have

$$
\frac{(E(\Delta) f, g)}{\rho(\Delta)}=\frac{\rho_{0}(\Delta)}{\rho(\Delta)} \frac{(E(\Delta) f, g)}{\rho_{0}(\Delta)} .
$$

Again, we let $\Delta_{0}$ denote the $\rho_{0}$-null set (independent of $f, g \in \mathcal{H}_{+}$) where $d(E(\cdot) /, g) / d \rho_{0}$ fails to exist and introduce the $\rho$-null set $\Delta_{1}$ where $d \rho_{0} / d \rho$ fails to exist [15, Chapter 4, Theorem 15.7]. Then if $\lambda \notin \Delta_{0} \cup \Delta_{1}$, the assertions for $\rho$ are evident. Let $S \subset \Delta_{0}-\Delta_{1}$. Then $\rho_{0}(S)=0,(E(S) f, g)=0$ and by the Radon-Nykodym theorem

$$
\int_{S} \frac{d(E(\cdot) f, g)}{d \rho} d \rho=0
$$

implying $d(E(\cdot) /, g) / d \rho=0 \rho$-a.e. on $\Delta_{0}-\Delta_{1}$; that is $d E(\cdot) f / d \rho=0 \rho$-a.e. on $\Delta_{0}-\Delta_{1}$. The proof is complete.

Berezanskir gives several examples of suitable spaces $H_{+}$and operators $T$ 
for the case when $\mathcal{H}_{0}=L_{2}(G)$, where $G$ is a region in $E^{n}$, and $A$ is a selfadjoint operator on $G$. One of the simplest is to take $\mathcal{H}_{+} \subseteq W_{2}^{l}(G)(l>n / 2)$ and $(u, v)_{+}=(q u, q v)_{l, 2}$ where $q$ is a suitably chosen real valued function.

The next theorem establishes an abstract pre-Hilbert space of solutions to $A \phi=\lambda \phi$ where $A=\int \lambda E(d \lambda)$. Let $\mathbb{M}_{\lambda}=\left\{\phi_{\lambda, f}: f \in \mathcal{H}_{+}\right\}$.

We shall be primarily interested in Theorem 2 for $\rho=\rho_{0}$, although it is proved here for any positive locally bounded Borel measure-i.e. one for which the various derivatives can be proved to exist.

Theorem 2. $\mathbb{M}_{\lambda}$ is a pre-Hilbert space with the inner product

$$
\langle\phi, \psi\rangle_{\lambda}=d(E(\cdot) f, g) / d \rho
$$

where $\phi, \psi$ are the weak derivatives $d E(\cdot) f / d \rho, d E(\cdot) g / d \rho$ resp.

Proof. We first show that the inner product is well defined.

Suppose $E(\Delta) f_{1} / \rho(\Delta) \rightarrow \phi$, and $E(\Delta) f_{2} / \rho(\Delta) \rightarrow \phi$ as $\Delta$ shrinks to $\{\lambda\}$; then $E(\Delta)\left(f_{1}-f_{2}\right) / \rho(\Delta) \rightarrow 0$. It follows from the inequality $|(E(\Delta) /, g)| \leq$ $(E(\Delta) f, f)^{1 / 2}(E(\Delta) g, g)^{1 / 2}$ that $\left(E(\Delta)\left(f_{1}-f_{2}\right), g\right) / \rho(\Delta) \rightarrow 0$. Consequently,

$$
\begin{aligned}
\left(E(\Delta) f_{1}, g_{1}\right) & / \rho(\Lambda)-\left(E(\Delta) f_{2}, g_{2}\right) / \rho(\Lambda) \\
& =\left(E(\Delta)\left(f_{1}-f_{2}\right), g_{1}\right) / \rho(\Lambda)+\left(f_{2}, E(\Delta)\left(g_{1}-g_{2}\right)\right) / \rho(\Delta) \\
& \rightarrow 0 \text { as } \Delta \text { shrinks to }\{\lambda\}
\end{aligned}
$$

and therefore the inner product is well defined. Conjugate linearity for $\langle\cdot, \cdot\rangle_{\lambda}$ follows easily from the conjugate linearity of the original inner product $(\cdot, \cdot)$.

Now we prove that $\|\phi\|_{\lambda}=0$ implies $\phi=0$.

Suppose $\langle\phi, \phi\rangle_{\lambda}=0$; that is $(E(\Delta) /, f) / \rho(\Delta) \rightarrow 0$. But $|(E(\Delta) f, g)| \leq$ $(E(\Delta) f, f)^{1 / 2}(E(\Delta) g, g)^{1 / 2}$. Hence $(E(\Delta) f, g) / \rho(\Delta) \rightarrow 0$ on a dense set of $g-s$ and therefore for all $g \in \mathcal{H}_{-}$.

One is led naturally to ask whether the norms $\|\cdot\|_{-\rightarrow}\|\cdot\|_{\lambda}$ are comparable when restricted to $\pi_{\lambda}$. In answer to this question we show in Corollary 2 that $\left\|\phi_{\lambda, f}\right\|_{-} \leq C\left\|\phi_{\lambda, f}\right\|_{\lambda}$ and in the example at the end (also studied in [1, p. 345]) that the opposite inequality is not possible.

A further study of $M_{\lambda}$ requires some machinery. If $E(\cdot)$ is a spectral measure in $\mathcal{H}$ and $f \in \mathcal{H}$ we define

$$
E-\operatorname{span}(f)=\operatorname{clospan}\{E(\Lambda) f: \Lambda \text { ranges over Borel sets }\}
$$

where clospan $\equiv$ closed span. We shall say that $\left\{g_{n}\right\}$ is an $E$-basis for $\mathcal{H}$ if $\left(E(\Delta) g_{n}, g_{m}\right)=0$ for all Borel sets $\Delta, m \neq n$, and

$$
\sum_{n} \bigoplus E-\operatorname{span}\left(g_{n}\right)=J \text {. }
$$


Such a basis exists if and only if $\mathcal{H}$ is separable.

It may be necessary henceforth to work with a sequence whose nonzero members constitute an orthonormal set. We call such a sequence practically orthonormal.

We shall also require the following result:

Lemma 1. If $\sigma$ is an additive Borel set function, $\rho$ is a positive locally bounded Borel measure and $A$ is any Borel set sucb that, for $\lambda \in A$, $\overline{\lim }_{\Delta \rightarrow \lambda} \sigma(\Delta) / \rho(\Delta) \geq a$. Then $\sigma(A) \geq a \rho(A)$.

Proof. The proof uses a standard technique [16, Chapter IV, Lemma 15.4] together with a suitable Vitali Covering Theorem [11].

The next two theorems are fundamental.

Theorem 3. Let $\left\{g_{n}\right\} \subset \mathcal{H}_{+}$be an E-basis in $\mathcal{H}_{0^{\circ}}$ Then for $\rho$-almost all $\lambda$ we can conclude that the elements $\psi_{n} \in \mathbb{M}_{\lambda}$ defined by

$$
\psi_{n}= \begin{cases}\phi_{\lambda, g_{n}} /\left\|\phi_{\lambda, g_{n}}\right\|_{\lambda} & \text { if } \phi_{\lambda, g_{n}} \neq 0, \\ 0 & \text { otherwise }\end{cases}
$$

satisfy $\Sigma_{n}\left\|\psi_{n}\right\|_{-}^{2}<\infty$ and $\left(\psi_{n}, \psi_{m}\right)_{\lambda}=0$ for $m \neq n$.

Proof. By definition we have, for $\lambda \notin \Delta_{0}$,

$$
\left\|E(\Delta) g_{n} / \rho(\Delta)-\phi_{\lambda, g_{n}}\right\|-\rightarrow 0
$$

and

$$
\left\|E(\Delta) g_{n}\right\|^{2} / \rho(\Delta) \rightarrow\left\|\phi_{\lambda, B_{n}}\right\|_{\lambda}^{2}
$$

as $\Delta$ shrinks to $\{\lambda\}$. It follows that

$$
\left\|E(\Delta) g_{n} / \sqrt{\rho(\Delta)}\right\| E(\Delta) g_{n}\left\|-\psi_{n}\right\|-\rightarrow 0
$$

Let $b_{n, \Delta}=E(\Delta) g_{n} /\left\|E(\Delta) g_{n}\right\|, n=1,2, \ldots$. It is clear that $\left\{b_{n, \Delta}\right\}$ is practically orthonormal for every $\Delta$.

Since $T$ is a Hilbert-Schmidt operator we note that

$$
\left.\sum_{n=1}^{\infty}\left\|T b_{n, 1}\right\|^{2} \leq \| T E(\lrcorner\right)\left\|_{\mathrm{S}}^{2} \leq\right\| T \|_{\mathrm{S}}^{2}
$$

(the subscript $S$ denoting Schmidt norm).

Let $\sigma$ be defined on Borel sets by

$$
\sigma(\Delta)=\|T E(\Delta)\|_{\mathrm{S}}^{2}
$$


To see that $\sigma$ is additive it is sufficient to observe that, if $\Delta_{1} \cap \Delta_{2}=\phi$ and $\left\{e_{n}\right\},\left\{f_{n}\right\}$ are complete orthonormal sets for $E\left(\Delta_{1}\right) \mathcal{H}, E\left(\Delta_{2}\right) \mathcal{H}$ resp., then $\left\{e_{n}\right\} \cup$ $\left\{f_{n}\right\}$ is a complete orthonormal set for $E\left(\Delta_{1} \cup \Delta_{2}\right) \mathcal{H}$.

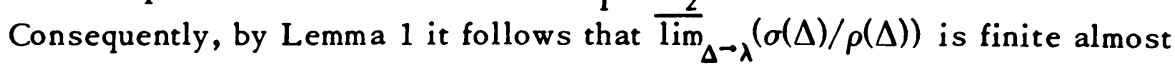
every where. Since

$$
\sum_{n=1}^{\infty} \lim _{\Delta \rightarrow \lambda} \frac{\left\|T b_{n, \Delta}\right\|^{2}}{\rho(\Delta)} \leq \varlimsup_{\Delta \rightarrow \lambda} \frac{\sigma(\Delta)}{\rho(\Delta)}
$$

and

$$
\lim _{\Delta \rightarrow \lambda} \frac{\left\|T b_{n, \Delta}\right\|^{2}}{\rho(\Delta)}=\left\|\psi_{n}\right\|_{-}^{2}
$$

the first conclusion of the theorem follows. For the second conclusion we note that, if $n \neq m$,

$$
\left\|\phi_{\lambda, g_{n}}\right\|_{\lambda}\left\|\phi_{\lambda, g_{m}}\right\|_{\lambda}\left(\psi_{n}, \psi_{m}\right)_{\lambda}=\lim _{\Delta \rightarrow \lambda} \frac{\left(E(\Delta) g_{n^{\prime}} g_{m}\right)}{\rho(\Delta)}=0,
$$

which completes the proof.

Corollary 1. If $\left\{g_{n}\right\} \subseteq \mathcal{H}_{+}$is an E-basis for $\mathcal{H}_{0}$, then the Hilbert space $H_{\lambda}$ spanned by $\left\{\phi_{\lambda, g_{n}}\right\}$, that is the completion of span $\left\{\phi_{\lambda, g_{n}}\right\}$, is contained in $\mathcal{H}_{-}$.

Proof. From Theorem 3 we have that the $\psi_{n}$ defined by

$$
\psi_{n}=\phi_{\lambda, 8_{n}} /\left\|\phi_{\lambda, 8_{n}}\right\|_{\lambda}, \quad n=1,2, \cdots,
$$

constitute a complete orthonormal set in $H_{\lambda}$ and that $\Sigma\left\|\psi_{n}\right\|_{-}^{2}<\infty$. If $\psi \in H_{\lambda}$ then $\psi=\Sigma_{n=1}^{\infty} C_{n} \psi_{n}$ where $\sum_{n=1}^{\infty}\left|C_{n}\right|^{2}<\infty$. We conclude that

$$
\|\psi\|_{-} \leq \sum_{n=1}^{\infty}\left|C_{n}\right|\left\|\psi_{n}\right\|_{-} \leq\left(\sum_{n=1}^{\infty}\left|C_{n}\right|^{2}\right)^{1 / 2}\left(\sum_{n=1}^{\infty}\left\|\psi_{n}\right\|_{-}^{2}\right)^{1 / 2}<\infty,
$$

whence $\psi \in \mathcal{H}_{\text {. }}$

So far we have been able to prove existence of $\phi_{\lambda, g_{n}} \rho$-a.e. as well as some of its properties for any given positive locally bounded Borel measure $\rho$. If we wish to prove completeness of the generalized eigenfunction expansion, however, $\rho$ must give nonzero weight to subsets of the spectrum of $A=\int \lambda E(d \lambda)$; that is $\rho_{0}=\operatorname{tr}\left(T^{*} E(\cdot) T\right)$ must be absolutely continuous with respect to $\rho$. To avoid encumbering the formulas we shall simply assume in the Completeness Theorem that $\rho=\rho_{0}$.

Theorem 4. If $\rho=\rho_{0}, f \in \mathcal{H}_{+}$and $\left\{g_{n}\right\} \subseteq \mathcal{H}_{+}$is an E-basis for $\mathcal{H}_{0}$, then for 
$\rho_{0}$-almost every value of $\lambda$ there exists a sequence $\left\{C_{n}(\lambda)\right\}$ of complex numbers such that

$$
\phi_{\lambda, f}=\sum_{n=1}^{\infty} C_{n}(\lambda) \phi_{\lambda, 8_{n}}
$$

with convergence in the $\|\cdot\|_{\lambda}$ sense.

Proof. Since we have already shown that $\pi_{\lambda}$ is a pre-Hilbert space it suffices to prove that

$$
\left\|\phi_{\lambda, f}\right\|_{\lambda}=\sum_{n=1}^{\infty}\left|C_{n}(\lambda)\right|^{2}\left\|\phi_{\lambda, 8 n}\right\|_{\lambda}^{2} \rho_{0} \text {-a.e. } \lambda .
$$

Since $\left\{g_{n}\right\}$ is an $E$-basis we have

$$
f=\sum_{n=1}^{\infty} \int C_{n}(\lambda) E(d \lambda) g_{n},
$$

and it follows that

$$
\|E(\Delta) f\|^{2}=\sum_{n=1}^{\infty} \int_{\Delta}\left\|C_{n}(\lambda)\right\|^{2}\left(E(d \lambda) g_{n}, g_{n}\right) .
$$

Since $(E(\cdot) f, f)$ is absolutely continuous with respect to $\rho_{0}(\cdot)$ for all $f \in \mathcal{H}_{+}$,

$$
\|f\|^{2}=\int \frac{d}{d \rho_{0}}\left(E(\lambda) f_{0} f\right) \rho_{0}(d \lambda) \quad \forall f \in \mathscr{H}_{+} .
$$

If we note, furthermore, that

$$
\lim _{\Delta \rightarrow \lambda} \frac{(E(\Delta) f, f)}{\rho_{0}(\Delta)}=\frac{d(E(\cdot) f, f)}{d \rho_{0}}(\lambda)=\left\|\phi_{\lambda, f}\right\|_{\lambda},
$$

it is clear that (2) with $\Delta=(-\infty, \infty)$ becomes

$$
\int\left\|\phi_{\lambda, f}\right\|_{\lambda}^{2} \rho_{0}(d \lambda)=\sum_{n=1}^{\infty} \int\left|C_{n}(\lambda)\right|^{2}\left\|\phi_{\lambda, g_{n}}\right\|_{\lambda}^{2} \rho_{0}(d \lambda) .
$$

If we divide (2) by $\rho(\Delta)$ and take the limit as $\Delta$ shrinks to $\{\lambda\}$ we deduce that $\rho_{0}$-a.e. $\lambda$

$$
\left\|\phi_{\lambda, f}\right\|_{\lambda}^{2} \geq \sum_{n=1}^{\infty}\left|C_{n}(\lambda)\right|^{2}\left\|\phi_{\lambda, g_{n}}\right\|_{\lambda}^{2}
$$

Combining (3) and (4) we deduce (1). This completes the proof. At this point let us define $\pi_{\lambda}$ to be the $\|\cdot\|_{\lambda}$-closure of $\pi_{\lambda}$. Then we have 
Corollary 2. There exists a constant $C$ such that

$$
\|\phi\|_{-} \leq C\|\phi\|_{\lambda} \text { for all } \phi \in \Re_{\lambda} \text {. }
$$

Proof. By Corollary 1 and Theorem 4 we conclude that $\pi_{\lambda} \subseteq \mathcal{H}_{-}$. The identity mapping from $\pi_{\lambda}$ into $\mathcal{H}_{-}$is certainly closed, so our result follows by the Closed Graph Theorem.

Remark 1. Corollary 2 has, of course, an obvious direct proof which al so yields

$$
C=\left(\sum_{n}\left\|\psi_{n}\right\|^{2}\right)^{1 / 2}
$$

We now return to the study of $\pi_{\lambda}$ as a space of generalized eigenvectors for $A=\int \lambda E(d \lambda)$.

Theorem 5. The elements $\phi \in \Re_{\lambda}$ satisfy $A \phi=\lambda \phi$ in the sense that $\left(\phi,(\Lambda-\lambda)_{0}\right)_{0}=0$ for all $u$ such that both $u$ and $A u$ belong to $\mathcal{H}_{+}$.

Proof. We prove the result first for $\phi \in \mathbb{M}_{\lambda}$. Then we have

$$
(\phi,(A-\lambda) u)=\lim _{\Delta \rightarrow \lambda} \frac{(E(\Lambda) f,(\Lambda-\lambda) u)}{\rho(\Lambda)} .
$$

Since

$$
E(\lambda)(A-\lambda) u:=\int_{\Delta}(\mu-\lambda) E(d \mu) u
$$

we conclude

$$
\|E(\lambda)(\Lambda-\lambda) u\| \leq \operatorname{diam}(\lambda)\|E(\lambda) u\| .
$$

Consequently,

$$
\frac{\left|\left(E(, \lambda) f_{0}(\Lambda-\lambda) u\right)\right|}{\rho(\lambda)} \leq \operatorname{diam}(\lambda)\left[\frac{(E(\lambda) f \cdot f)}{\rho(\Lambda)}\right]^{1 / 2}\left[\frac{(E(\lambda) u, u)}{\rho(\lambda)}\right]^{1 / 2}
$$

which tends to 0 as $\Delta \rightarrow \lambda$. Now let $\phi \in \Re_{\lambda}$ and $\left\{\phi_{n}\right\} \subset M_{\lambda}$ such that $\left\|\phi-\phi_{n}\right\|_{\lambda} \rightarrow 0$. Then, by Corollary 2, $\left\|\phi-\phi_{n}\right\|_{-} \rightarrow 0$. But since we have

$$
|(\phi,(\Lambda-\lambda) u)| \leq\left\|\phi-\phi_{n}\right\|_{-}\|(\Lambda-\lambda) u\|_{+}+\left|\left(\phi_{n},(\Lambda-\lambda) u\right)\right|,
$$

the proof is complete.

Remark 2. The preceding proof shows that $(\phi,(A-\lambda) u)=0$ al so on the $\|\cdot\|_{-}$-closure of $\pi_{\lambda}$. The example at the end shows that the $\|\cdot\|_{-}$-closure of $\pi_{\lambda}$ can be strictly larger than $\pi_{\lambda}$. Consequently, the condition $(\phi,(\Lambda-\lambda) u)=0$ is not sufficient to determine the generalized eigenfunctions $\phi \in \mathcal{r}_{\lambda}$. 
3. Generalized eigenfunctions and resolvent limits. In practice when we deal with a differential operator, the preceding theory cannot be applied unless we know a considerable amount about its spectral measure from other sources. Since the spectral measure is usually the unknown, whereas the resolvent and its kernel are more accessible, we show that the Hilbert space $\pi_{\lambda}$ can be obtained al so by taking limits of the resolvent.

In order to accomplish this we shall make use of the following generalization of a well-known result [15, p. 224] whose proof follows Theorem 9.

Lemma 2. Let $\mu$ be any Borel measure on $\mathbf{R}$ satisfying $\int\left[|\mu|(d x) /\left(1+x^{2}\right)\right]$ $<\infty(|\mu|$ is the variation of $\mu)$ and $\rho$ be a nonnegative Borel measure on $\mathbf{R}$ satisfying $\int\left[\rho(d x) /\left(1+x^{2}\right)\right]<\infty$. Then

$$
\lim _{\epsilon \rightarrow 0^{+}} \frac{\int\left[\epsilon /\left((x-y)^{2}+\epsilon^{2}\right)\right] \mu(d y)}{\int\left[\epsilon /\left((x-y)^{2}+\epsilon^{2}\right)\right] \rho(d y)}=\frac{d \mu}{d \rho}(x) \quad \rho-a . e .
$$

An application of this lemma yields our basic theorem.

Theorem 6. Let $E(\cdot)$ be a spectral measure, $A=\int \lambda E(d \lambda), R_{z}=(A-z)^{-1}$, and let $\rho$ satisfy the conditions of Lemma 2. Then, for $\rho$-a.e. $\lambda$ and $f \in \mathcal{H}_{+}$,

$$
\operatorname{wk}_{\epsilon \rightarrow 0^{+}} \frac{\left(R_{\lambda-i \epsilon}-R_{\lambda+i \epsilon}\right) f}{\int\left[2 i \epsilon \rho(d \mu) /\left((\lambda-\mu)^{2}+\epsilon^{2}\right)\right]}=\lim _{\Delta \rightarrow \lambda} \frac{E(\Delta) f}{\rho(\Delta)} .
$$

(Both sides in the above equality must be considered as functionals in $\mathcal{H}_{\text {- with }}$ domain $\mathcal{H}_{+}$)

Proof. Certainly $(E(\cdot) f, g)$ satisfies the conditions for $\mu$ in Lemma 2. Furthermore, for any $f, g \in \mathcal{H}_{0}$,

$$
\frac{\left(\left(R_{\lambda-i \epsilon}-R_{\lambda+i \epsilon}\right) f, g\right)}{\int\left[2 i \epsilon \rho(d \mu) /\left((\lambda-\mu)^{2}+\epsilon^{2}\right)\right]}=\frac{\int\left[\epsilon(E(d \mu) f, g) /\left((\lambda-\mu)^{2}+\epsilon^{2}\right)\right]}{\int\left[\epsilon \rho(d \mu) /\left((\lambda-\mu)^{2}+\epsilon^{2}\right)\right]},
$$

so that by Lemma 2 we conclude

$$
\lim _{\epsilon \rightarrow 0+} \frac{\left(\left(R_{\lambda-i \epsilon}-R_{\lambda+i \epsilon}\right) f, g\right)}{\int\left[2 i \epsilon \rho(d \mu) /\left((\lambda-\mu)^{2}+\epsilon^{2}\right)\right]}=\lim _{\Delta \rightarrow \lambda} \frac{(E(\Delta) f, g)}{\rho(\Delta)} \rho \text {-a.e. } \lambda \text {. }
$$

Since we already know from Theorem 1 that, for $f, g \in \mathcal{H}_{+}$, wk $\lim _{\Delta \rightarrow \lambda}(E(\Delta) f / \rho(\Delta))=\phi_{\lambda, f} \in \mathcal{H}_{-}$and $\lim _{\Delta \rightarrow \lambda}((E(\Delta) f, g) / \rho(\Delta))=\phi_{\lambda, f}(g)$, the proof is complete.

Remark 3. If $\rho_{0}(\Delta)=\operatorname{tr}\left(T^{*} E(\Delta) T\right)$ and $T$ is as in Theorem 1 , then we have the estimate $|(E(\Delta) f, g)| \leq\|f\|_{+}\|g\|_{+} \rho_{0}(\Delta)$ and it follows that

$$
\int \frac{\epsilon}{(\lambda-\mu)^{2}+\epsilon^{2}}(E(d \mu) f, g) \leq\|f\|+\|g\|+\int \frac{\epsilon}{(\lambda-\mu)^{2}+\epsilon^{2}} \rho_{0}(d \mu) \text {. }
$$


In the case of general $\rho$ we still have

$$
\left|\frac{\left(\left(R_{\lambda-i \epsilon}-R_{\lambda+i \epsilon}\right) f_{,} g\right)}{\int\left[2 i \epsilon \rho(d \mu) /\left((\lambda-\mu)^{2}+\epsilon^{2}\right)\right]}\right| \leq C\|f\|_{+}\|g\|_{+} \rho \text {-a.e. } \lambda
$$

where $C$ is independent of $f_{,} g$ but does depend on $\lambda, \mu$ and has a finite limit as $\mu \rightarrow 0+$.

Although Theorem 6 is stated for fairly general $\rho$, it is still more useful to use the measure $\rho_{0}$ especially if completeness of the eigenfunction expansion is desired. The next theorem gives a formula showing that $\rho_{0}$ can in fact be easily computed.

Theorem 7. Let $\left\{e_{n}\right\}$ be a complete orthonormal set in $\mathcal{H}_{0}$ and $f_{n}=T e_{n}$, $n=1,2, \ldots$. Then

$$
\int \frac{2 i \epsilon \rho_{0}(d \mu)}{(\lambda-\mu)^{2}+\epsilon^{2}}=\sum_{n=1}^{\infty}\left(\left(R_{\lambda-i \epsilon}-R_{\lambda+i \epsilon}\right) f_{n}, f_{n}\right) .
$$

Proof. We have

$$
\begin{aligned}
\left(\left(R_{\lambda-i \epsilon}-R_{\lambda+i \epsilon}\right) f_{n^{\prime}} f_{n}\right) & =\int \frac{\epsilon}{(\mu-\lambda)^{2}+\epsilon^{2}}\left(E(d \lambda) f_{n^{\prime}} f_{n}\right) \\
& =\int \frac{\epsilon}{(\mu-\lambda)^{2}+\epsilon^{2}}\left(T^{*} E(d \lambda) T e_{n^{\prime}} e_{n}\right) .
\end{aligned}
$$

Hence

$$
\begin{aligned}
\sum_{n=1}^{\infty}\left(\left(R_{\lambda-i \epsilon}-R_{\lambda+i \epsilon}\right) f_{n}, f_{n}\right) & =\sum_{n=1}^{\infty} \frac{2 i \epsilon}{(\mu-\lambda)^{2}+\epsilon^{2}}\left(T^{*} E(d \lambda) T e_{n^{\prime}} e_{n}\right) \\
& =\int \frac{\epsilon}{(\mu-\lambda)^{2}+\epsilon^{2}} \sum_{n=1}^{\infty}\left(T^{*} E(d \lambda) T e_{n^{\prime}} e_{n}\right)
\end{aligned}
$$

where the interchange of summation and integration is justified because both the measures and integrand are positive. This completes the proof of the theorem.

4. Eigenfunction expansion. We now state and prove a theorem showing how an eigenfunction expansion can be obtained from any sufficiently large family of generalized eigenfunctions.

Theorem 8. Let $\{\phi(\lambda, \alpha): \lambda \in \mathbf{R}, a=1,2, \ldots\}$ be a family of elements from

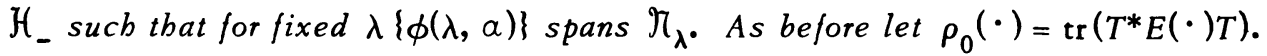
Then there exists a matrix valued function $\left\{\sigma_{\alpha \beta}(\lambda)\right\}$ sucb that, for all $1 . g$ $\epsilon \mathfrak{H}_{+}$

$$
(f, g)=\int \rho_{0}(d \lambda) \sum_{a} \sum_{\beta}(f, \phi(\lambda, \alpha))_{0} \sigma_{a \beta}(\lambda) \overline{(g, \phi(\lambda, \beta))_{0}}
$$



have

Proof. Since $(E(\cdot) f, g)$ is absolutely continuous with respect to $\rho_{0}(\cdot)$ we

$$
(f, g)_{0}=\int \rho_{0}(d \lambda) \frac{d}{d \rho_{0}}(E(\cdot) f, g)_{0}=\int \rho_{0}(d \lambda)\left(\phi_{\lambda, f}, g\right)_{0}
$$

where $\phi_{\lambda, f} \in \Re_{\lambda}$ whenever $f_{0} g \in \mathcal{H}_{+}$. For each real $\lambda$ we choose a basis $\left\{\phi(\lambda, \alpha): \alpha \in I_{\lambda}\right\} \subset\{\phi(\lambda, \alpha)\}$. Since clospan $\{\phi(\lambda, a)\}=\pi_{\lambda}$, we can find unique constants $C_{a}(f), \alpha \in I_{\lambda}$, such that

$$
\dot{\varphi}_{\lambda, f}=\sum_{\alpha \in I_{\lambda}} C_{\alpha}(f) \phi(\lambda, \alpha)
$$

with convergence in the $\|\cdot\|_{\lambda}$ norm. It is easy to see that $\left\{\phi(\lambda, \beta): \beta \in I_{\lambda}\right.$, $\beta \neq \alpha\}^{\perp}$ is a one dimensional subspace and contains an element $\phi^{*}(\lambda, \alpha)$ such that $\left\langle\phi(\lambda, \alpha), \phi^{*}(\lambda, \alpha)\right\rangle=1$. In fact $\left\{\phi^{*}(\lambda, \alpha)\right\}$ is a dual basis satisfying

$$
\left\langle\phi(\lambda, \alpha), \phi^{*}(\lambda, \beta)\right\rangle=\delta_{a \beta}
$$

( $\delta_{\alpha \beta}$ being the Kronecker delta). From (6) and (7) we conclude that

$$
C_{a}(f)=\left\langle\phi_{\lambda, f}, \phi^{*}(\lambda, a)\right\rangle \text {. }
$$

Suppose now we write each $\phi^{*}$ in terms of the $\phi$ basis, that is

$$
\phi^{*}(\lambda, \alpha)=\sum \overline{\sigma_{a \beta}}(\lambda) \phi(\lambda, \beta),
$$

and substitute in to (9) obtaining

$$
C_{\alpha}(f)=\sum_{\beta} \sigma_{\alpha \beta}(\lambda)\left\langle\phi_{\lambda, f}, \phi(\lambda, \beta)\right\rangle .
$$

If we recall the definition of $\langle\cdot, \cdot\rangle$ it is clear that

$$
\left\langle\phi_{\lambda, f}, \phi(\lambda, \beta)\right\rangle=(f, \phi(\lambda, \beta))_{0}
$$

Certainly from (7) we have

$$
\left(\phi_{\lambda, f}, g\right)_{0}=\sum_{a \in I_{\lambda}} C_{a}(f)(\phi(\lambda, \alpha), g)_{0} .
$$

If we now combine (10), (11) and (12), and substitute into (6) we obtain the theorem with the sums being taken over $\alpha, \beta \in I_{\lambda}$. This, however, completes the proof if we let $a_{\sigma \beta}=0$ for $(\alpha, \beta) \notin I_{\lambda} \times I_{\lambda}$.

Remark 4. If the $\phi(\lambda, \alpha)$ fail to be measurable then the set $\Lambda_{a}=\left\{\lambda: \alpha \in I_{\lambda}\right\}$ or the functions $(f, \phi(\lambda, a))_{0}, \sigma_{a_{\beta}}(\lambda)$ could fail to be measurable. The conclusion of Theorem 8 is not affected because $\Sigma_{a} \Sigma_{\beta}(f, \phi(\lambda, \alpha)), \sigma_{a_{\beta}}(\lambda) \overline{(g, \phi(\lambda, \beta))_{0}}=$ $\left(d / d \rho_{0}\right)(E(\cdot) f, g)$ is always measurable. If the hypothesis of Theorem 8 is bolstered by the assumption that, for each $\alpha, \phi(\lambda, \alpha)$ be weakly measurable, then the $I_{\lambda}$ can be chosen to yield measurability of $\Lambda_{a},\left(f_{0} \phi(\lambda, a)\right)_{0}$, and $\sigma_{a_{\beta}}(\lambda)$. 
An effective choice strategy is as follows: For each fixed $\lambda$ delete each element in the list $\phi(\lambda, 1), \phi(\lambda, 2), \ldots$ which happens to be dependent on its predecessors, starting from the beginning and working up. Then the set $I_{\lambda}$ of remaining indices yields the required measurability properties.

5. Pointwise limits of the resolvent. Finally, we recall the well-known result from the theory of a selfadjoint differential operator $A$ that the pointwise limits

$$
\lim _{\epsilon \rightarrow 0^{+}}\left(R_{\lambda-i \epsilon}-R_{\lambda+i \epsilon}\right) f
$$

exist whenever $f$ is in the absolutely continuous subspace corresponding to $A$. It is obvious that such a limit fails to exist when $f$ is in the eigenspace of $A$. Lemma 2, however, enables us to obtain a straightforward generalization of this result from the theorem of Garding [9].

Theorem 9. Let $A$ be a selfadjoint extension of the formally selfadjoint elliptic partial differential operator $L$ acting on a domain $G$ in $E^{n}$. Then, if $f$ is a function of compact support in $G$ and $\rho$ is a locally bounded Borel measure, there exists a set $\Lambda_{0}$ with $\rho\left(\Lambda_{0}\right)=0$ such that

$$
\lim _{\epsilon \rightarrow 0+} \frac{\left[\left(R_{\lambda-i \epsilon}-R_{\lambda+i \epsilon}\right) f\right](x)}{\int\left[2 i \epsilon \rho(d \mu) /\left((\lambda-\mu)^{2}+\epsilon^{2}\right)\right]}
$$

exists for $x \in G$ and $\lambda \notin \Lambda_{0}$.

Proof. From Garding's theorem we have

$$
\begin{aligned}
{\left[\left(R_{\lambda-i \epsilon}-R_{\lambda+i \epsilon}\right) f\right](x) } & =\int_{G} d y\left(\int \tau(d \lambda) \frac{2 i \epsilon}{(\mu-\lambda)^{2}+\epsilon^{2}} \theta(x, y, \lambda)\right) f(y) \\
& =\int r(d \lambda) \frac{2 i \epsilon}{(\mu-\lambda)^{2}+\epsilon^{2}}\left(\int_{G} \theta(x, y, \lambda) f(y) d y\right)
\end{aligned}
$$

where $\theta(\cdot, \cdot, \lambda) \in C^{m}(G \times G)$ r-a.e. $\lambda, m$ being the order of $L$, is integrable on compact subsets of $G \times G \times \mathbf{R}$, and

$$
L_{x} \theta(x, y, \lambda)=L_{y} \theta(x, y, \lambda)=\lambda \theta(x, y, \lambda) .
$$

If we let $\int_{G} \theta(x, y, \lambda) f(y) d y=b(x, \lambda)$, then

$$
L_{x} b(x, \lambda)=\lambda b(x, y) \text {. }
$$

Let $G_{0}$ be a compact subdomain of $G$ and $m(\lambda)=\sup _{x \in \Omega_{0}}|b(x, \lambda)|, b_{1}(x, \lambda)=$ $b(x, \lambda) / m(\lambda)$. Then (14) becomes

$$
\left[\left(R_{\lambda-i \epsilon}-R_{\lambda+i \epsilon}\right) f\right](x)=\int \frac{2 \dot{i \epsilon}}{(\lambda-\mu)^{2}+\epsilon^{2}} b_{1}(x, \lambda)_{m}(\lambda)_{r}(d \lambda) .
$$


Since $L_{x} b_{1}(x, \lambda)=\lambda b_{1}(x, \lambda)$ where $L$ is elliptic and $\left|b_{1}(x, \lambda)\right| \leq 1$, it follows from the elliptic estimates that $b_{1}$ will satisfy a Lipschitz condition in $x \in G_{0}$ with constant independent of $\lambda$. Furthermore, using Lemma 2, we can show that there exists a set $\Lambda_{0}$ of $m(\lambda) \tau(d \lambda)$ measure zero such that

$$
\lim _{\epsilon \rightarrow 0^{+}} \frac{\left[\left(R_{\lambda-i \epsilon}-R_{\lambda+i \epsilon}\right) f\right](x)}{\int\left[2 i \epsilon m(\lambda) \tau(d \lambda) /\left((\lambda-\mu)^{2}+\epsilon^{2}\right)\right]}
$$

exists on a countable dense set of $x$ in $G_{0}$. The Lipschitz condition is then used to extend the result to all $x \in G_{0}$. The conclusion of the theorem now follows from another application of Lemma 2 to the ratio

$$
\frac{\int\left[2 i \epsilon m(\lambda) \tau(d \lambda) /\left((\lambda-\mu)^{2}+\epsilon^{2}\right)\right]}{\int\left[2 i \epsilon \rho(d \lambda) /\left((\lambda-\mu)^{2}+\epsilon^{2}\right)\right]} .
$$

Proof of Lemma 2. It suffices to prove the result for the positive and negative parts of the real and imaginary parts of $\mu$ separately. Hence we shall assume in the proof that $\mu$ is also a positive measure. It is known that $(d \mu / d \rho)(x)$ exists for $\rho$-a.e. $x$ as a limit of quotients (see preamble to Theorem 1). We prove in fact that ( 5 ) holds whenever the derivative exists, and in order to convey the idea of the proof with a minimum of notation, we take $x=0$.

Let $b(\epsilon, y)=\epsilon /\left(y^{2}+\epsilon^{2}\right)$. Then if $\delta>0$,

$$
\frac{\int b(\epsilon, y) \mu(d y)}{\int h(\epsilon, y) \rho(d y)}=\frac{\left(\int_{-\delta}^{\delta}+\int|y| \geq \delta\right) b(\epsilon, y) \mu(d y)}{\int b(\epsilon, y) \rho(d y)}
$$

which we denote in the remainder of the proof by $Q(\epsilon)$.

The starting point for the proof is the integration by parts formula

$$
\int_{\Delta_{\delta}} b(\epsilon, y) \mu(d y)=b(\epsilon, \delta) \mu\left(\Delta_{\delta}\right)+\int_{0}^{\delta} \mu\left(\Delta_{s}\right) b^{\prime}(\epsilon, s) d s
$$

where $\Delta_{s}=[-s, s]$ and $b^{\prime}(\epsilon, s)=-(\partial b / \partial s)(\epsilon, s) \geq 0$, which can be proved by techniques similar to those of [15, p. 225, Lemma 11.9].

Suppose now that $(d \mu / d \rho)(0)=C$. Given $C^{\prime}<C$ and $C^{\prime \prime}>C$ we choose $\delta$ such that $0<s<\delta$ implies $C^{\prime} \rho\left(\Delta_{s}\right)<\mu\left(\Delta_{s}\right)<C^{\prime \prime} \rho\left(\Delta_{s}\right)$. Then

$$
C^{\prime} \int_{0}^{\delta} \rho\left(\Delta_{s}\right) b_{1}(\epsilon, s) d s<\int_{0}^{\delta} \mu\left(\Delta_{s}\right) b_{1}(\epsilon, s) d s<C^{\prime \prime} \int_{0}^{\delta} \rho\left(\Delta_{s}\right) b_{1}(\epsilon, s) d s .
$$

If we now combine (18) and (19) with (17) and rearrange terms we find that $Q(\epsilon)$ is bounded below by

$$
\begin{aligned}
& B\left(C^{\prime}, \epsilon\right)=C^{\prime} \\
& +\frac{\int|y| \geq \delta}{b(\epsilon, y) \mu(d y)+b(\epsilon, \delta) \mu\left(\Delta_{\delta}\right)-C^{\prime}\left[\int_{|y| \geq \delta} b(\epsilon, y) \rho(d y)+b(\epsilon, \delta) \rho\left(\Delta_{\delta}\right)\right]}
\end{aligned}
$$


and above by $B\left(C^{\prime \prime}, \epsilon\right)$. We know from Lemma 3 that the denominators above vanish only on a set of $\rho$-measure zero. Furthermore, $\lim _{\epsilon \rightarrow 0+} B(C, \epsilon)=0$ so that for $\epsilon$ small enough we have $C^{\prime}<Q(\epsilon)<C^{\prime \prime}$. Since $C^{\prime}$, $C^{\prime \prime}$ was subject only to the condition $C^{\prime}<C<C^{\prime \prime}$, it follows that $\lim _{\epsilon \rightarrow 0+} Q(\epsilon)=C$.

Lemma 3. If $\rho$ is a positive Borel measure on the real line for which $\int\left[\rho(d x) /\left(1+x^{2}\right)\right]<\infty$, then the set of points

$$
\left\{x:{\underset{\lim }{\epsilon \rightarrow 0}+} \int \frac{\epsilon}{(x-y)^{2}+\epsilon^{2}} \rho(d y)=0\right\}
$$

bas a $\rho$-measure zero.

Proof. Since

$$
\int \frac{\epsilon}{(x-y)^{2}+\epsilon^{2}} \rho(d y) \geq \int_{|x-y| \leq \epsilon} \frac{\epsilon}{(x-y)^{2}+\epsilon^{2}} \rho(d y) \geq \frac{1}{2 \epsilon} \rho([x-\epsilon, x+\epsilon]),
$$

it suffices to show that the lower derivative vanishes on a $\rho$-null set.

Let $N$ be the set

$$
N=\left\{x: \underset{b \rightarrow 0^{+}}{\lim } \frac{\rho([x-b, x+b])}{2 b}=0\right\} .
$$

Given $\epsilon>0$ and $x \in N$ there exists a sequence $\left\{b_{k}\right\}$ decreasing to 0 such that $\rho\left(J_{x, k}\right)<2 \epsilon b_{k}$, where $J_{x_{0}, k}=\left[x-b_{k^{\prime}} x+b_{k}\right]$. Then $g=\left\{J_{x, k}\right\}$ is a Vitali covering for $N$, and by the Vitali Covering Theorem there exists a disjoint subsequence $\left\{J_{k}\right\} \subset \mathcal{G}$ such that $\rho\left(N-\bigcup_{k} J_{k}\right)=0$. Then $\rho\left(J_{k}\right)<2 b_{k} \epsilon$, and $\rho\left(\cup_{k} J_{k}\right) \leq(b-a)_{\epsilon}$, which implies $\rho(N)=0$.

Example. We show in this example that $\boldsymbol{r}_{\lambda}$ can be strictly smaller than $\mathcal{H}_{-}$.

Let $\mathcal{H}_{0}=L_{2}\left(E^{2}\right)$ and let $q(x)=\left(1+\left|x_{1}\right|\right)^{1+\epsilon}\left(1+\left|x_{2}\right|\right)^{1+\epsilon}$ where $\epsilon>0$. We define $T^{-1}$ by $\left(T^{-1} u\right)(x)=q(x)\left(\partial^{2} u / \partial x_{1} \partial x_{2}\right)(x)$ whence

$$
T f(x)=\int K(x, \xi) f(\xi) d \xi,
$$

where

$$
K(x, \xi)=\chi_{S(\xi)}(x) \operatorname{sgn}\left(\xi_{1}\right) \operatorname{sgn}\left(\xi_{2}\right) / q(\xi),
$$

$S(\xi)$ being the square with diagonally opposed corners at the origin and at $\xi$ respectively. That is, if we let $I\left(x_{j}\right)$ denote $\int_{x_{j}}^{\infty} d \xi_{j}$ for $x_{j}>0$ and $-\int_{-\infty}^{x_{j}} d \xi_{j}$ for $x_{j}<0$, we find

$$
T f(x)=I\left(x_{1}\right) I\left(x_{2}\right)(f(\xi) / q(\xi)) .
$$

It is shown in [1] that $T$ is a Hilbert-Schmidt operator.

Suppose now that $E$ is the spectral measure corresponding to multiplication 
by $x_{1}$, i.e., for any Borel set $\Delta$,

$$
(E(\Lambda) f)(x)=\chi_{\Delta}\left(x_{1}\right) f(x) .
$$

In order to compute the measure $\rho_{0}$ we consider the operator $T^{*} E(\Delta) T$ whose kernel is $\int d y \overline{K(y, x)} \chi_{\Delta}\left(y_{1}\right) K(y, x)$. We restrict our attention to the diagonal values and compute

$$
\int d y \chi_{\Delta}\left(y_{1}\right)|K(y, x)|^{2}=\int d y \chi_{\Delta}\left(y_{1}\right)\left|\frac{\chi_{S}(x)}{q(x)}\right|^{2} .
$$

Then

$$
\begin{aligned}
\rho_{0}(\Delta) & =\operatorname{tr}\left(T^{*} E(\Delta) T\right)=\int d x d y \chi_{\Delta}\left(y_{1}\right)\left|\frac{\chi_{S}(x)}{q(x)}\right|^{2} \\
& =\int_{\Delta} d y_{1} \int d y_{2} \int d x\left|\frac{\chi_{S(x)}(y)}{q(x)}\right|^{2}=\int_{\Delta} \sigma\left(y_{1}\right) d y_{1},
\end{aligned}
$$

where

$$
\sigma\left(y_{1}\right)=\int d y_{2} \int d x\left|\frac{\chi_{S(x)}(y)}{q(x)}\right|^{2} .
$$

Thus, if $f \in \mathcal{H}_{+}$,

$$
\phi_{\lambda, f}(x)=\lim _{\Delta \rightarrow \lambda} \frac{E(\Delta) f(x)}{\int_{\Delta} \sigma\left(x_{1}\right) d x_{1}}=\frac{\delta\left(x_{1}-\lambda\right) f\left(\lambda, x_{2}\right)}{\sigma(\lambda)},
$$

where $\delta$ is a delta function at the origin. Futhermore,

(20)

$$
\begin{aligned}
\left\|\phi_{\lambda, f}\right\|_{\lambda}^{2} & =\lim _{\Delta \rightarrow x_{1}} \frac{\|E(\Delta) f\|^{2}}{\int_{\Delta} \sigma\left(x_{1}\right) d x_{1}} \\
& =\lim _{\Delta \rightarrow \lambda} \frac{\int_{\Delta} d x_{1} \int d x_{2}|f(x)|^{2}}{\int_{\Delta} \sigma\left(x_{1}\right) d x_{1}}=\frac{\int\left|f\left(\lambda, x_{2}\right)\right|^{2} d x_{2}}{\sigma(\lambda)} .
\end{aligned}
$$

If we assume in addition that $f$ has support in the first quadrant and $\lambda>0$, then

$$
\left(T \phi_{\lambda, f}\right)(x)=\left\{\begin{array}{l}
\int_{x_{1}}^{\infty} d \xi_{1} \int_{x_{2}}^{\infty} d \xi_{2} \frac{\delta\left(\xi_{1}-\lambda\right) f\left(\lambda, \xi_{2}\right)}{\sigma(\lambda)\left(1+\left|\xi_{1}\right|\right)^{1+\epsilon}\left(1+\left|\xi_{2}\right|\right)^{1+\epsilon}}, \quad x_{1}, x_{2}>0 \\
0 \quad \text { otherwise, }
\end{array}\right.
$$




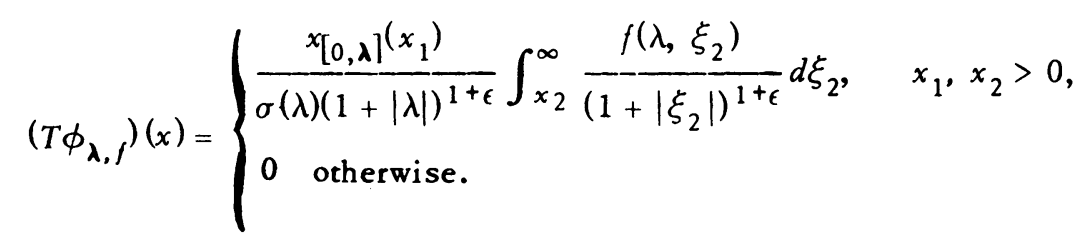

Hence,

(21) $\left\|\phi_{\lambda, f}\right\|_{-}^{2}=\left\|T \phi_{\lambda, f}\right\|_{0}^{2}=\frac{\lambda}{\sigma^{2}(\lambda)(1+|\lambda|)^{2+2 \epsilon}} \int_{0}^{\infty} d x_{2}\left|\int_{x_{2}}^{\infty} \frac{f\left(\lambda_{2} \xi_{2}\right)}{\left(1+\left|\xi_{2}\right|\right)^{1+\epsilon}} d \xi_{2}\right|^{2}$.

If we now let $f\left(x_{2}\right)=1$ for $x_{2}>0$ and 0 for $x_{2}<0$ and take $\epsilon>1 / 2$, we find that $\left\|\phi_{f, \lambda}\right\|_{\lambda}=\infty$ but

$$
\left\|\phi_{\lambda_{,} f}\right\|_{-}^{2}=\frac{\lambda}{\sigma(\lambda)^{2}(1+|\lambda|)^{2+2 \epsilon}} \int_{0}^{\infty} \frac{1}{\epsilon^{2}} \frac{1}{\left(1+x_{2}\right)^{2 \epsilon}} d x_{2}<\infty
$$

which is what we intended to show.

It is an easy matter in this example to prove a special case of Corollary 2. We proceed as follows. From (21) we have

$$
\begin{aligned}
\left\|\phi_{\lambda, f}\right\|_{-}^{2} & =\frac{\lambda}{\sigma^{2}(\lambda)(1+|\lambda|)^{2+2 \epsilon}} \int_{0}^{\infty} d x_{2} \int_{x_{2}}^{\infty} d \xi_{2} \int_{x_{2}}^{\infty} d \xi_{2}^{\prime} \frac{f\left(\lambda_{2}, \xi_{2}\right) f\left(\lambda, \xi_{2}^{\prime}\right)}{\left(1+\left|\xi_{2}\right|\right)^{1+\epsilon}\left(1+\left|\xi_{2}^{\prime}\right|\right)^{1+\epsilon}} \\
& =\frac{\lambda}{\sigma^{2}(\lambda)(1+|\lambda|)^{2+2 \epsilon}} \int_{0}^{\infty} d \xi_{2} \int_{0}^{\infty} d \epsilon_{2}^{\prime} \frac{\min \left(\xi_{2}, \xi_{2}^{\prime}\right) f\left(\lambda, \xi_{2}\right) f\left(\lambda, \xi_{2}^{\prime}\right)}{\left(1+\left|\xi_{2}\right|\right)^{1+\epsilon}\left(1+\left|\xi_{2}^{\prime}\right|\right)^{1+\epsilon}}
\end{aligned}
$$

If we observe that $\min \left(\xi_{2}, \xi_{2}\right) \leq \sqrt{\xi_{2} \xi_{2}}$ we find the last expression becomes

$$
\begin{aligned}
\left\|\phi_{\lambda, f}\right\|_{-}^{2} & \leq \frac{\lambda}{\sigma^{2}(\lambda)(1+|\lambda|)^{1+\epsilon}}\left(\int_{0}^{\infty} d \xi_{2} \frac{\sqrt{\xi_{2}} f\left(\lambda, \xi_{2}\right)}{\left(1+\left|\xi_{2}\right|\right)^{1+\epsilon}}\right) \\
& \leq \frac{\lambda}{\sigma^{2}(\lambda)(1+|\lambda|)^{1+\epsilon}}\left(\int_{0}^{\infty} \frac{\xi_{2} d \xi_{2}}{\left(1+\left|\xi_{2}\right|\right)^{2+2 \epsilon}}\right)\left(\int_{0}^{\infty} \mid f\left(\lambda,\left.\xi_{2}\right|^{2} d \xi_{2}\right)\right.
\end{aligned}
$$

from which we conclude that

$$
\left\|\phi_{\lambda, f}\right\|_{-}^{2} \leq\left(\lambda / \sigma(\lambda)(1+|\lambda|)^{2+2 \epsilon}\right)\left\|\phi_{\lambda, f}\right\|_{\lambda}^{2} .
$$

If $f$ does not have support in the first quadrant then certainly it can be written as $f=f_{1}+f_{2}+f_{3}+f_{4}$ with $f_{i}$ having support in the $i$ th quadrant. An inequality of the above type can be deduced for each $f_{i}$ and then shown to hold for the sum. It follows then that

$$
\left\|\phi_{\lambda, f}\right\|_{-}^{2} \leq C\left\|\phi_{\lambda, f}\right\|_{\lambda}^{2}
$$

for all $f \in \mathcal{H}_{+}$. 


\section{REFERENCES}

1. Ju. M. BerezanskiY, Expansion in eigenfunctions of selfadjoint operators, "Naukova Dumka", Kiev, 1965; English transl., Transl. Math. Monographs, vol. 17, Amer. Math. Soc., Providence, R. I., 1968. MR 36 \#5768; \#5769.

2.—. Spaces with negative norm, Uspehi Mat. Nauk 18 (1963), no. 1 (109), 63-96 = Russian Math. Surveys 18 (1963), no. 1, 63-95. MR 29 \#1553.

3.- On an eigenfunction expansion for selfadjoint operators, Ukrain. Mat. Ž. 11 (1959), 16-24; English transl., Amer. Math. Soc. Transl. (2) 93 (1970), 227-238. MR 23 \#A518; MR 42 \#4.

4.- Eigen function expansions of selfadjoint operators, Mat. Sb. 43 (85) (1957), 75-126. (Russian) MR 21 \#3646.

5. F. E. Browder, Eigenfunction expansions for formally self-adjoint partial differ. ential operators. I, II, Proc. Nat. Acad. Sci. U. S. A. 42 (1956), 769-771, 870-872. MR 19, 1061.

6.- The eigenfunction expansion theorem for the general self-adjoint singular elliptic partial differential operator. I. II, Proc. Nat. Acad. Sci. U. S. A. 40 (1954), 454463. MR 16, 134.

7. N. Dunford and J. T. Schwartz, Linear operators. I: General theory, Pure and Appl. Math., vol. 7, Interscience, New York, 1958. MR 22 \#8302; Vol. II: Spectral theory. Selfadjoint operators in Hilbert space, Interscience, New York, 1963. MR 32 \#6181.

8. Lars Gårding, Eigenfunction expansions, Partial Differential Equations (Proc. Summer Seminar, Boulder, Col., 1957), Interscience, New York, 1964, pp. 301-325. MR 29 \#2525.

9.—. Eigenfunction expansions connected with elliptic differential operators, Tolfte Skandinaviska Matematikerkongressen, Lund, 1953, pp. 44-55. MR 17, 158.

10. E. Gerlach, On spectral representation for selfadjoint operators. Expansion in generalized eigenelements, Ann. Inst. Fourier (Grenoble) 15 (1965), fasc. 2, 537-574. MR $32 \# 8172$.

11. M. de Guzmán, A covering lemma with applications to differentiability of measures and singular integral operators, Studia Math. 34 (1970), 299-317. MR 41 \#621.

12. G. I. Kac, Spectral decompositions of self-adjoint operators in terms of general. ized elements of a Hilbert space, Ukrain. Math. Ž. 13 (1961) no. 4, 13-33. (Russian) MR $26 \# 1764$.

13. - Expansion in characteristic functions of self-adjoint operators, Dokl. Akad. Nauk SSSR 119 (1958), 19-22. (Russian) MR 21 \#3647.

14. T. Kato, Perturbation theory for linear operators, Die Grundlehren der math. Wissenschaften, Band 132, Springer-Verlag, New York, 1966. MR 34 \#3324.

15. W. Rudin, Real and complex analysis, McGraw-Hill, New York, 1966. MR 35 \#1420.

16. S. Saks, Théorie de l'intégrale, Monografie Mat., vol. 7, PWN, Warsaw, 1937.

DEPARTMENT OF MATHEMATICS, UNIVERSITY OF TORONTO, TORONTO 181, ONTARIO, CANADA 